\title{
Strangelet spectra from type II supernovae
}

\author{
H. Vucetich* \\ Facultad de Ciencias Astronómicas e Geofísicas, Universidad Nacional de La Plata, Paseo del Bosque S/N, (1900) La Plata, Argentina \\ J. E. Horvath \\ Instituto Astronômico e Geofísico, Universidade de São Paulo, Avenida Miguel Stéfano 4200, Água Funda, 04301-904, São Paulo, Brasil
}

(Received 5 September 1997; published 4 May 1998)

\begin{abstract}
We study in this work the fate of strangelets injected as a contamination in the tail of a "strange matterdriven" supernova shock. A simple model for the fragmentation and braking of the strangelets, when they pass through the expanding oxygen shell is presented and solved to understand the reprocessing of this component. We find that the escaping spectrum is a scaled-down version of the one injected at the base of the oxygen shell. The supernova source is likely to produce low-energy particles of $A \sim 100-1000$ quite independently of the initial conditions. However, it is difficult that ultrarrelativistic strangelets (such as the hypothetical Centauro primaries) can have an origin in those explosive events.
\end{abstract}

[S0556-2821(98)00412-3]

PACS number(s): 96.40.De, 12.39.-x, 97.60.Bw

\section{INTRODUCTION}

More than a decade ago a celebrated paper by Witten [1] (see also the previous works by Bodmer [2] and Chin and Kerman [3]) suggested that we may have been overlooking the true ground state of hadronic interactions. According to this work, strange matter (cold catalyzed quark matter nearly symmetric in $u, d$ and $s$ flavors) would offer a possible form of bypassing the limitations imposed by the Pauli principle in ordinary matter because of the existence of a third (strange) Fermi sea to share the energy of the system. Then, the energy per baryonic number unit would be smaller when compared to two-flavor quark matter and if this reduction is large enough, strange matter created after a weak-interaction time scale may be the lowest energy state. Following this suggestion, which was essentially based on a bulk Fermi gas picture, the properties of strange matter and the droplet version, termed strangelets, were investigated [4-6]. Particular attention has been paid to the possible shell structure in the fewquark strangelets [7-9], which are the ones expected to show up in heavy-ion collision experiments [10]. A great deal of papers also explored astrophysical scenarios which could render a non-zero interstellar medium (ISM) abundance of strangelets (which would cause all neutron stars to become strange stars) $[1,11-14]$, the latter point being specially important because of the criticisms $[15,16]$ raised to the strange quark matter (SQM) hypothesis based on pulsar glitch observations, to which the basic strange star models [17] have no reasonable explanation to offer (see Refs. [18] for more elaborated stellar models possibly containing an explanation of that data).

Concerning the experimental detection at the Earth, it is interesting to note that, even before the official "invention", of SQM by Witten, some cosmic ray events [20] were tentatively associated with quark blobs primaries [21]. A sum-

*Affiliated with CONICET. mary of the reported SQM candidates in several experiments is shown in Table I.

In Refs. [11,12,14,25,26] aspects of SQM production have been investigated and discussed. One particularly puzzling aspect of all candidates is their relatively low baryonic number $A$. Calculations indicate [4-6] that SQM tends to be more tightly bound for increasing $A$, so that injection of favored SQM fragments $\geqslant 10^{5} \mathrm{amu}$ in any astrophysical event would require substantial reprocessing to get down to $\sim 10^{2}$ amu strangelets. The authors of Ref. [25] have estimated the reprocessing time-scales by using model spallation cross sections of fragments of $\Delta$ amu with $H$ and $O$ of the form

$$
\sigma(\Delta)=\sigma_{0}\left(\frac{m}{m_{0}}\right)^{2 / 3} \exp \left(-\Delta / \Delta_{0}\right),
$$

where $\Delta_{0}$ is the preferred emitted cluster; and found that reprocessing is very ineffective, unless the strangelets can pass through a very dense oxygen shell. Although this situation is very unlikely for the popular double-degenerate coalescence scenario $[1,12]$; it is precisely the situation expected in strange matter-driven supernovae scenarios $[27,28]$. In the latter a second shock arises because of the exothermic transition $n \rightarrow u d s+e n e r g y$ and is expected to carry a contamination of strangelets in the low-velocity tail as a byproduct of turbulent mixing $[11,28]$. Ejection of the strangelets with $v \sim 0.1 c$ is one of the possible mechanisms for a non-zero ISM abundance and thus competes with the coalescence events. The relative frequencies of both phenomena

TABLE I. SQM event candidates.

\begin{tabular}{cccc}
\hline \hline$Z$ & A & E/nuc $(\mathrm{GeV})$ & Ref. \\
\hline- & 75 & $\sim 1000$ & {$[20]$} \\
$20-40$ & - & $\geqslant 2.22$ & {$[22]$} \\
14 & 400 & 0.45 & {$[23]$} \\
46 & $\geqslant 1000$ & - & {$[24]$} \\
\hline \hline
\end{tabular}


$\sim 10^{-2} \mathrm{yr}^{-1}$ and $\sim 10^{-4} \mathrm{yr}^{-1}$, respectively, would be enough to identify the dominating source, if the total mass ejected in strangelets could be calculated. However, since we lack reliable estimates of the latter, we have tried to infer the mass working backwards from the reported events [14]. We have obtained $10^{-6} M_{\odot}$ and $3 \times 10^{-13} M_{\odot}$ by normalizing the flux to the events reported in Ref. [23] and Centauros [20], respectively. However, these results refer strictly to the abundance of SQM primaries with $A \sim 100$ measured at the Earth, and therefore the question of the most likely "isotope" escaping from whatever source to the ISM still remains.

\section{SPALLATION OF STRANGELETS BY OXYGEN}

Let us address the specific case of SQM supernova ejection. As explained above, the basic picture postulates of a (mildly relativistic to non-relativistic) strangelet gas travelling in the tail of the secondary shock, which encounters the expanding oxygen shell. According to Ref. [29], the expansion of the dense oxygen may be modelled by a number density evolution of the form $n(t)=n_{0} \exp \left(-t / \tau_{\text {exp }}\right)$, where $\tau_{\text {exp }} \simeq 0.1 \mathrm{~s}$ is a multiple of the free-fall time-scale appropriate for that physical situation. The (heavy) strangelets encountering oxygen targets will loose energy and baryon number, a process that may be described in the hydrodynamical approximation by the following set of coupled equations

$$
\begin{aligned}
\frac{d m}{d t} & =-\int d \Delta n(t) \sigma(\Delta) v \\
m \frac{d v}{d t} & =-C \frac{\pi}{2} m_{o x} n(t) v^{2} R_{0}^{2}\left(\frac{m}{m_{0}}\right)^{2 / 3}+\dot{m} \xi v,
\end{aligned}
$$

where $v$ is the velocity of the strangelets relative to the expanding oxygen shell, $m_{o x}$ is the mass of an oxygen nucleus, $R_{0}$ is the reference radius corresponding to an $A=200$ strangelet (taken to be $5.8 \mathrm{fm}$ ) and $C$ is the von Karman constant. We note that the r.h.s. of Eq. (2.2) has been integrated over the fragment distribution and that we have included in the parameter $0<\xi<1$ the details of the transfer in the reaction $A+{ }^{16} \mathrm{O} \rightarrow A^{\prime}+{ }^{16} \mathrm{O}^{\prime}+\Delta+$ energy.

Note that we have neglected the absorption of oxygen nuclei by the strangelet. These fusion reactions can be crudely described by a geometric cross-section times the well-known Gamow factor. A simple calculation shows that the quotient of the fusion to the spallation cross-sections is $\sim \exp \left(-32 \pi \alpha A^{1 / 3} c v^{-1}\right)$, where $\alpha$ is the fine structure constant and the approximation $Z \sim 2 A^{1 / 3}$ has been made [19]. Absorption is thus suppressed by a large factor and is never important in the process we are considering.

Eqs. (2.1) and (2.2) can be combined into a single differential equation for the momentum loss which has the solution

$$
v=v_{i}\left(\frac{m}{m_{i}}\right)^{D-1}
$$

where $v_{i}$ and $m_{i}$ are the initial velocity and mass of the strangelet and $D=\left(\pi C m_{o x} R_{0}^{2}\right) /\left(2 \Delta_{0} \sigma_{0}\right)+\xi$. Going back to Eqs. (2.1) and (2.2), we obtain the evolution of the strangelet mass with time as

$$
m=m_{i}\left[1-\left(\frac{4}{3}-D\right) \frac{\tau_{\text {exp }}}{\tau_{i}} \frac{\Delta_{0}}{m_{i}} f(t)\right]^{3 /(4-3 D)},
$$

with $\tau_{i}=\left(n_{0} v_{i} \sigma\right)^{-1}$ the initial mean-free path of the strangelets in the oxygen shell and $f(t)=\left[1-\exp \left(-t / \tau_{\text {exp }}\right)\right]$.

Finally, we find using Eqs. (2.3) and (2.4) the kinetic energy of the strangelet,

$$
E_{K}=\left(\frac{m}{m_{i}}\right)^{2 D-1} E_{K, i}
$$

and therefore the total energy of the strangelet $E_{T}$ from $E_{T}^{2}$ $=m^{2}+E_{K}^{2}$.

\section{REGIMES OF FRAGMENTATION}

An inspection of Eqs. (2.3), (2.4) and (2.5) reveals that there are different regimes of fragmentation separated by the value of the exponent $D$. In all the four cases to be discussed below, the mass of the strangelet decreases (i.e. spallation occurs) irrespective of the specific value of $D$; until the available kinetic energy in the center-of-mass frame $E_{K}^{c . m}$. is not enough to strip a chunk whick is bound by an amount $\Delta_{0} E_{b} / m_{p}\left(E_{b} \sim 10 \mathrm{MeV}\right.$ is the binding energy per baryon number unit in the strangelet). The strangelet will survive as long as the latter condition can be reached before the mass is driven to zero (or, more precisely, to a value below $m_{\text {min }}$ corresponding to the minimum stable baryon number of the strange matter $\left.A_{\min }\right)$. The possible regimes are

\section{A. $0<D<1 / 2$}

If $D$ belongs to this range, Eqs. (2.3) and (2.5) show that both the kinetic energy and velocity grow with time. It follows immediately that the strangelets can never satisfy the mass freezeout condition imposed on $E_{K}^{c . m}$. because there is always enough energy available to power the spallations. Thus, the particles in this regime evaporate completely and do not constitute an astrophysically interesting case.

\section{B. $1 / 2<D<1$}

In this case the kinetic energy decreases although the velocity increases with time. However, in the center-of-mass frame the kinetic energy $E_{K}^{c . m}$. scales approximately as

$$
E_{K}^{c . m .}=E_{i, o x}\left(\frac{m}{m_{i}}\right)^{2(D-1)}
$$

(with $E_{i, o x}$ the initial kinetic energy of the oxygen) and cannot decrease either. Therefore the particles also evaporate in this regime. 


\section{C. $1<D<4 / 3$}

Now both the kinetic energy and the velocity of the strangelets decrease with time. Spallation proceeds until the point in which [see Eq. (3.1)] the freezout condition is reached

$$
E_{i, o x}\left(\frac{m}{m_{i}}\right)^{2(D-1)}=\frac{\Delta_{0}}{m_{p}} E_{b} .
$$

This regime gives rise to a scaling law for the mass of the form

$$
\frac{m_{F}}{m_{i}}=\left(\frac{\Delta_{0}}{m_{p}} \frac{E_{b}}{E_{i, o x}}\right)^{1 / 2(D-1)},
$$

$m_{F}$ being the final or freezeout mass of the surviving strangelet.

\section{D. $D>4 / 3$}

The situation is essentially the same as in the former point, but now the mass decreases slowly than before [see Eq. (2.4)]. This does not lead to any substantial change in the picture because the time scale $\tau_{i} \simeq 10^{-16} \mathrm{~s}$ is so short that the strangelets would not be able to travel large distances before freezeout sets in or the oxygen shell expands substantially.

\section{DISCUSSION}

From the above expressions the full emerging spectrum in mass and energy could be computed for a given injection spectrum at the source. Even without performing a detailed computation some general features are apparent. First of all Eqs. (2.3)-(2.5) show the dependence of the results with the parameter $D$, which are dramatically different depending on its actual value. Recalling the definition and using $C=0.5$ as measured in the case of subsonic spheres in laboratory, we have found a strict lower bound of $D \geqslant 0.75$ by setting $\xi$ $=0$. Thus, it is quite likely that the physical situation corresponds to the third and fourth cases above. An important corollary is that the escaping spectrum of strangelets is a scaled-down version of the one injected by the secondary shock. A second major point is that the stripped fragments may or may not decay depending on the (unknown) actual value of the minimum stable strangelet $A_{\text {min }}$. If $A_{\text {min }} \sim 10$, these fragments are likely to survive further spallation because of their non-relativistic character. On the other hand, they would quickly decay into ordinary hadrons if $A_{\min }$ $\sim 100$. In any case the spallation interactions of the ejected strangelets with oxygen are so frequent in the expanding shell that the final distribution will be concentrated around the lowest stable "isotope" and near the minimum (spallation cutoff) energy, although it is likely that further braking occurs either in the shell or in the ISM. For example, encounters of the escaping strangelets with giant molecular clouds are quite frequent and may help to strip a few baryon number units. Finally, we note that is difficult in this scenario to obtain relativistic strangelets such as the ones required to fit the primaries of the Centauro events. The natural outcome from supernovae would be non-relativistic primaries in the range $A \sim 100-1000$; which are easily obtained from these events if the strangelets injected initially by the shock have $10^{5}-10^{8}$ amu for $v_{i} \leqslant 0.1 c$ [see Eq. (2.4)]. A more realistic treatment of the initial strangelet spectrum requires a model of fragmentation of the (initially homogeneous) strange matter fluid into strangelets in a turbulent environment [28]; even though we expect the mass scaling law Eq. (3.3) also to hold in this case. This subject will be discussed in a future work.

It is very important to measure the actual flux of candidates to connect it with the details of the source and, through simulations like the one in Ref. [14], to measure the total mass in the galaxy (for example, a recent experiment seems to have measured a much lower flux than the one determined in [23]). Further experimental and theoretical studies would be helpful to address the relativistic ejection of strangelets and the form of the spectra for each astrophysical scenario. Work on these subjects is in progress and will be reported elsewhere.

\section{ACKNOWLEDGMENTS}

We would like to acknowledge the financial support of the CNPq (Brazil) and SECYT (Argentina) to J.E.H. and H.V, respectively. The PROINTER Program of São Paulo University has provided financial support to maintain this collaboration.
[1] E. Witten, Phys. Rev. D 30, 272 (1984).

[2] A. R. Bodmer, Phys. Rev. D 4, 1601 (1971).

[3] S. A. Chin and A. K. Kerman, Phys. Rev. Lett. 43, 1292 (1979).

[4] E. Farhi and R. L. Jaffe, Phys. Rev. D 30, 2379 (1984).

[5] M. S. Berger and R. L. Jaffe, Phys. Rev. C 35, 213 (1987).

[6] J. Madsen, in Strange and Quark Matter, edited by G. Vassiliadis et al. (World Scientific, Singapore, 1995), p. 191; Phys. Rev. D 47, 5156 (1993).

[7] K. Takahashi and R. N. Boyd, Astrophys. J. 327, 1009 (1988).

[8] F. C. Michel, Phys. Rev. Lett. 60, 677 (1988).

[9] E. P. Gilson and R. L. Jaffe, Phys. Rev. Lett. 71, 332 (1993).

[10] K. Pretzl et al., in Strange and Quark Matter, edited by G.
Vassiliadis et al. (World Scientific, Singapore, 1995), p. 230.

[11] O. G. Benvenuto and J. E. Horvath, Mod. Phys. Lett. A 4, 1085 (1989).

[12] N. K. Glendenning, Mod. Phys. Lett. A 5, 2197 (1990).

[13] A. De Rújula and S. L. Glashow, Nature (London) 312, 734 (1984).

[14] G. A. Medina Tanco and J. E. Horvath, Astrophys. J. 464, 354 (1996).

[15] M. A. Alpar, Phys. Rev. Lett. 58, 2152 (1987).

[16] J. Madsen, Phys. Rev. Lett. 61, 2909 (1988).

[17] C. Alcock, E. Farhi and A. V. Olinto, Astrophys. J. 310, 261 (1986); P. Haensel, J. L. Zdunik, and R. Schaeffer, Astron. Astrophys. 160, 121 (1986); O. G. Benvenuto and J. E. Hor- 
vath, Mon. Not. R. Astron. Soc. 241, 43 (1989).

[18] O. G. Benvenuto, J. E. Horvath, and H. Vucetich, Phys. Rev. Lett. 64, 713 (1990); O. G. Benvenuto and J. E. Horvath, Mon. Not. R. Astron. Soc. 247, 584 (1990).

[19] H. Heiselberg, Phys. Rev. D 48, 1418 (1993).

[20] C. Lattes, Y. Fujimoto and S. Hagesawa, Phys. Rep. 65, 151 (1980).

[21] J. D. Bjorken and L. McLerran, Phys. Rev. D 20, 2353 (1979).

[22] P. B. Price et al., Phys. Rev. D 18, 1382 (1978).

[23] T. Saito et al., Phys. Rev. Lett. 65, 2094 (1990).
[24] M. Ichimura et al., Nuovo Cimento A 106, 84 (1993).

[25] T. Saito (unpublished).

[26] R. N. Boyd and T. Saito, Phys. Lett. B 298, 6 (1993).

[27] O. G. Benvenuto and J. E. Horvath, Phys. Rev. Lett. 63, 716 (1989).

[28] O. G. Benvenuto, J. E. Horvath and H. Vucetich, Int. J. Mod. Phys. A 4, 257 (1989); 6, 4769 (1991); H. Vucetich (unpublished).

[29] W. Fowler and F. Hoyle, Astrophys. J. 200, 345 (1964). 